\title{
Acosog
}


Country: USA

\section{Group: American College of Surgeons Oncology Group (ACOSOG)}

Chairs: $\quad$ D.M. Ota

Duke Clinical Research Institute/ACOSOG

2400 Pratt St., Terrace Level Room 0311

Durham, NC 27705

USA

Tel: +1919668 5987

Fax: + $19196687123 / 7156$

Email: David.ota@duke.edu

H. Nelson

Fred C. Anderson Professor of Surgery

Mayo Clinic College of Medicine

200 First St., SW

Rochester, MN 55905

USA

Tel: + 15072842511

Fax: +1 5072841794

Email: nelson.heidi@mayo.edu

Other

Subgroup

Head/

Member:

Other

Subgroup

Head/

Member:
Breast Committee

Chair Breast Organ Site Committee:

K.K. Hunt

Professor of Surgery, Chief Surgical Breast Section

The University of Texas M.D. Anderson Cancer Center

Chief Surgical Breast Section

Department of Surgical Oncology

1515 Holcombe Boulevard, Unit 444

Houston, TX 77030

USA

Tel: +17137927216

Fax: +17137924689

Email: khunt@mdanderson.org

Vice Chairs Breast Organ Site Committee:

A.M. Leitch

Professor of Surgery

University of Texas Southwestern Medical Center

5323 Harry Hines Blvd.

Dallas, TX 75390-9155

USA

Tel: + 12146483039

Fax: +1 2146488695

Email: marilyn.leitch@utsouthwestern.edu 
R.M. Simmons

A.K. and E.C. Weiskopf Associate Professor of Surgical Oncology Joan and Sanford Weill Medical College of Cornell University Department of Surgical Oncology The New York Presbyterian Hospital Iris Cantor Women's Health Center 425 East 61st St., 8th Floor New York, NY 10021 USA

Tel: +12128210870

Fax: +12128210832

Email: rms2002@med.cornell.edu

P.W. Whitworth

Vanderbilt University

Nashville Breast Center

Department of Surgery

Suite 401

300 20th Avenue North

Nashville, TN 37203

USA

Tel: +1 6152848229

Fax: +1 6152847794

Email: patwpatw@aol.com

Other Group Administrator:

Subgroup B. Martinez

Head/

Tel: +1919668 8451

Member: $\quad$ Email: marti025@notes.duke.edu

Other Clinical Trials Assistant:

Subgroup Susan Schwab

Head/ Tel: +1919668 8987

Member: $\quad$ Email: schwa009@notes.duke.edu

Website: $\quad$ www.acosog.org 
Title:

ACOSOG Z1031: A randomized phase III trial comparing 16-18 weeks of neoadjuvant exemestane $(25 \mathrm{mg}$ daily), letrozole $(2.5 \mathrm{mg})$, or anastrozole (1mg) in postmenopausal women with clinical stages II and III estrogen receptor positive breast cancer.

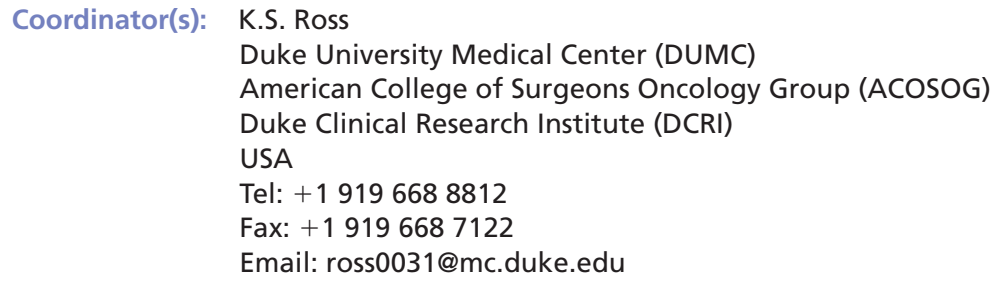

Principal

M.J. Ellis

Investigators:

Barnes Jewish Hospital

660 S. Euclid, Box 8056

St Louis, MO 63110

USA

Tel: +13143628866

Fax: +13143627086

Email: mellis@im.wustl.edu

\author{
J.A. Olson Jr \\ Duke University Medical Center \\ Department of Surgery \\ Box 3873 \\ Durham, NC 27710 \\ USA \\ Tel: +1 9196846523 \\ Fax: +19196816291 \\ Email: jaomd@duke.edu
}

Summary: $\quad$ - Opened in March 2006

- Target accrual: 375 patients

Primary Objective:

To determine whether anastrozole, exemestane or letrozole administered for 16-18 weeks as neoadjuvant endocrine treatment for postmenopausal patients with stage II or III ER + breast cancer should be chosen as the aromatase inhibitor of a future study that will compare a neoadjuvant aromatase inhibitor treatment with neoadjuvant chemotherapy.

Secondary Objectives:

- To compare the neoadjuvant treatment regimens relative to the rates of improvement in surgical outcome defined as follows: 
- For T4 a, b, c tumors: mastectomy with primary skin closure and negative surgical margins.

- For T3 and T2 tumors classified as requiring mastectomy at baseline: breast conserving surgery with negative final margins.

- For T2 tumors classified as potential candidates for breast conservation: wide excision at first attempt.

- To compare the radiological response rates (mammography and ultrasound by central radiological analysis) between these three neoadjuvant treatment regimens.

- To compare the relative safety of the neoadjuvant treatment regimens in terms of reported adverse events.

\section{Scheme:}

Z1031: A randomized phase III trial comparing 16-18 weeks of neoadjuvant exemestane ( $25 \mathrm{mg}$ daily), letrozole ( $2.5 \mathrm{mg}$ daily) or anastrozole (1 mg daily), in postmenopausal women with clinical stage II or III estrogen receptor postive breast cancer

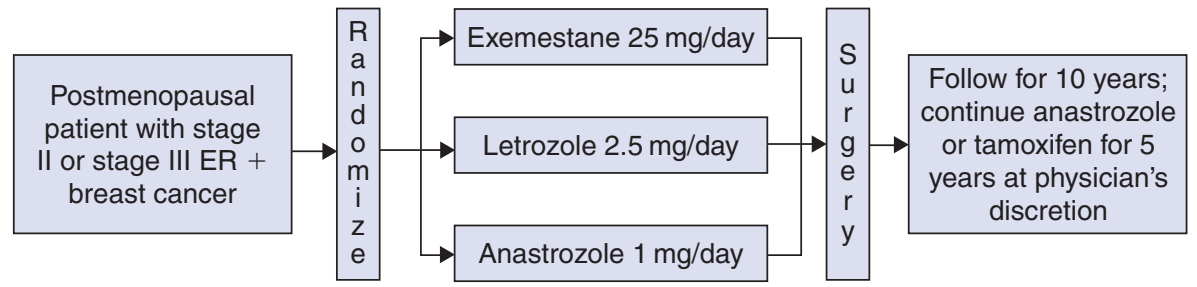

Update: $\quad$ Total accrual: 21 patients as of 25 September, 2006.

Related None available

Publications:

Topics: $\quad$ Aromatase inhibitors

- Breast conservative treatment

- Hormonal therapy

- Hormone receptor positive breast cancer

- Postmenopausal patients

Keywords: Neoadjuvant endocrine therapy, estrogen receptor positive breast cancer 


\section{Coordinator(s): K.S. Ross}

Duke University Medical Center (DUMC)

American College of Surgeons Oncology Group (ACOSOG)

Duke Clinical Research Institute (DCRI)

USA

Tel: +19196688812

Fax: +19196687122

Email: ross0031@mc.duke.edu

Principal

Investigator:
A.E. Giuliano

John Wayne Cancer Institute

2200 Santa Monica Boulevard, Suite 113

Santa Monica, CA 90404-2302

USA

Tel: +13108298089

Fax: +1 3109983995

Email:giulianoa@jwci.org

- Opened in May 1999

- Target accrual: 5300 patients

Primary and Secondary Objectives:

- To estimate the prevalence and to evaluate the prognostic significance of sentinel node micrometastases detected by immunohistochemistry (IHC).

- To estimate the prevalence and to evaluate the prognostic significance of bone marrow micrometastasis detected by immunocytochemistry (ICC).

- To evaluate the hazard rate for regional recurrence in women whose sentinel nodes are negative by hematoxylin and eosin (H\&E) staining.

- To provide a mechanism for identifying women whose sentinel nodes contain metastases detected by H\&E so that these women can be considered as candidates for Study Z0011. 


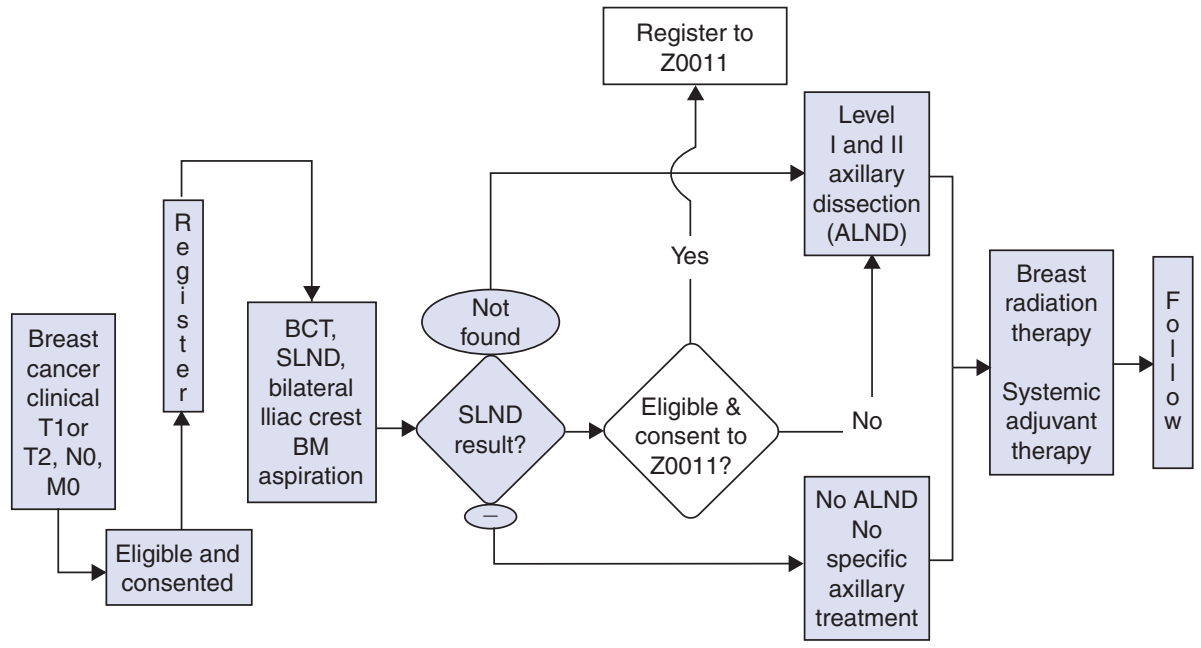

Update: - Study closed in May 2003 with 5539 patients.

- Five-year results have not yet been published.

Related Leitch AM, et al. Patterns of participation and successful

Publications: patient recruitment to ACOSOG Z0010, a phase II trial for patients with early stage breast cancer. Am J Surg 2005; 190(4): 539-542.

Posther KE, et al. Sentinel node skills verification and surgeon performance: data from a multicenter clinical trial for early stage breast cancer. Ann Surgery 2005; 242(4): 593-599; discussion 599-602.

Wilke LG, et al. Surgical complications associated with sentinel lymph node biopsy: results from a prospective international cooperative group trial. Ann Surg Oncol 2006; 13(4): 491-500.

Topics:
- Bone marrow micrometastasis
- - Loco-regional relapse
- Prognostic factors
- Sentinel node micrometastasis
- Sentinel node resection

Keywords: $\quad$ Sentinel lymph node biopsy, bone marrow micrometastases, immunohistochemistry 
Title:

ACOSOG Z0011: A randomized trial of axillary node dissection in women with clinical T1-2 N0 M0 breast cancer who have a positive sentinel node.

\author{
Coordinator(s): K.S. Ross \\ Duke University Medical Center (DUMC) \\ American College of Surgeons Oncology Group (ACOSOG) \\ Duke Clinical Research Institute (DCRI) \\ USA \\ Tel: +19196688812 \\ Fax: +19196687122 \\ Email: ross0031@mc.duke.edu
}

Principal

A.E. Giuliano

Investigator:

John Wayne Cancer Institute

2200 Santa Monica Boulevard, Suite 113

Santa Monica, CA 90404-2302

USA

Tel: +1 3108298089

Fax: +1 3109983995

Email: giulianoa@jwci.org

Summary: $\quad$ Opened in May 1999

- Target accrual: 1900 patients

Objectives:

- Long term: To assess whether overall survival for patients randomized to Arm 2 (no immediate axillary lymph node dissection, ALND) is essentially equivalent to (or better than) that for patients assigned to Arm 1 (completion ALND).

- Short term: To quantify and compare the surgical morbidities associated with sentinel lymph node dissection (SLND) plus ALND versus SLND alone.

Scheme:

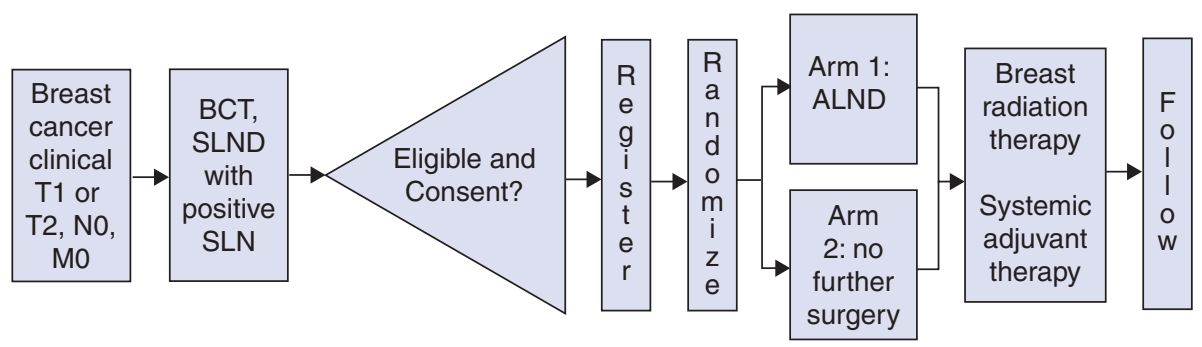


Update:

Related

Publications:

Topics:

Keywords:
- The study closed in 15 December 2004 secondary to slow accrual with 891 patients.

Lucci A, et al. Surgical complications associated with sentinel lymph node dissection (SLND) plus axillary lymph node dissection, versus SLND alone in the American College of Surgeons Oncology Group (ACOSOG) Trial Z0011. Ann Surg Oncol 2006; 13(2): 4.

- Axillary lymph node dissection

- Breast conservative treatment

- Loco-regional relapse

- Node positive breast cancer

- Sentinel node micrometastasis

- Sentinel node resection

Sentinel node biopsy, surgical morbidity, lymphedema 